\title{
RESPONSABILIDAD Y DIGNIDAD EN LA VIVIENDA ${ }^{1}$
}

\author{
Recibido 22 de Marzo de 2016 - Aceptado 15 de Mayo de 2016
}

\author{
Mauricio Javier Sierra Morales²
}

Universidad de la Gran Colombia. Bogotá, D.C., Colombia. mauricio.sierra@ugc.edu.co

Para citar este artículo / to reference this article:

Sierra, M. (2016). Responsabilidad y dignidad en la vivienda. Módulo Arquitectura CUC, Vol.17 N¹, 49-62.

\section{Resumen}

El presente texto responde a la pregunta, ¿Cuáles criterios y conceptos deberíamos, querríamos, y sobre todo, podríamos ser capaces de explicar, entender y transformar a fin de diseñar, y sobre todo, ejecutar eficaz y eficientemente una política pública de vivienda digna basada en criterios de responsabilidad social? Al intentar hacerlo, deja abierto otros interrogantes, como: ¿Además de la conocida triádica vitruviana, firmitas - utilitas - venustas, aplicable a los equipamientos colectivos de carácter comunitario, cuáles serían las bases contemporáneas para "otras" manifestaciones o creaciones arquitectónicas, tales como la vivienda, el espacio público e incluso, el paisaje como producto cultural de la distinción? El autor asume una postura epistemológica socio-crítica, basada en una metodología de investigación holística; sus principales hallazgos consisten en descubrir la delusión arquitectónica de la forma por la forma y en cómo utilizar la hermenéutica diatópica como antídoto a la misma. Por tanto, sus conclusiones están presentadas a manera de no-acuerdos, como punto de partida entre varios autores, el autor y el lector y su relevancia se basa en la posibilidad de restablecer la resiliencia y la dignidad como los nuevos criterios para definir la sustentabilidad, tanto de la forma como en el comportamiento de los seres humanos, y por supuesto, de su vivienda "digna" en un contexto de responsabilidad social.

\section{Palabras clave}

Dignitas, Restitutas, Sustentabilidad, Responsabilidad.

\footnotetext{
1 El presente trabajo de investigación pertenece al tema: ODS - Objetivos de Desarrollo Sostenible según ONU. Presentado en el IV Simposio Internacional de Responsabilidad Social de las Organizaciones (SIRSO) "Aportes teóricoprácticos para lograr los Objetivos de Desarrollo Sostenible en América Latina"; realizado por la Universidad de Santo Tomás (USTA) de Bogotá, los dias 29 y 30 de septiembre de 2016.
}

2 Postdoctorado Universidad Nacional de Córdoba; Doctorado Universidade Federal Do Rio De Janeiro; Maestría Universidad de los Andes. Pregrado Universidad Piloto de Colombia. 


\section{RESPONSIBILITY AND DIGNITY IN THE HOUSING}

\section{Abstract}

The present text responds to the question, What criteria and concepts should we, we would like, and above all, we could be able to explain, understand and transform in order to design, and above all, to execute a decent housing based public policy effectively and efficiently In criteria of social responsibility? When it tries to do so, it leaves open other questions, such as: In addition to the well - known Vitruvian triadic, firmitas - utilitas - venustas, applicable to collective community facilities, what would be the contemporary basis for "other" manifestations or architectural creations, such as Housing, public space and even the landscape as a cultural product of distinction? The author assumes a socio-critical epistemological stance, based on a holistic research methodology; Its main findings are to discover the architectural delusion of form by form and how to use diatopic hermeneutics as an antidote to it. Therefore, its conclusions are presented as non-agreements, as a starting point between several authors, the author and the reader and their relevance is based on the possibility of restoring resilience and dignity as the new criteria to define sustainability, Both in the form and behavior of human beings, and of course, in their "dignified" housing in a context of social responsibility.

\section{Keywords}

Dignitas, Restitutas, Sustainability, Responsibility. 
La fórmula que lleva a la responsabilidad social comienza con establecer una clara diferencia entre la forma y el comportamiento como categorías establecidas en la interrelación objeto - sujeto - omnijeto ${ }^{3}$ alrededor de la idea de sustentabilidad. Lo anterior redefine la idea original que tenemos del legado de la obra de Marco Vitruvio (15 A. de C.), acerca de la tríadica firmitas - utilitas - venustas, en el sentido que se requiere una actualización de los criterios que definen, o al menos se han constituido en la base o punto de partida de las áreas académicas en nuestras profesiones relacionadas a la construcción material y la habitabilidad edilicia desde la época antigua de la humanidad.

Tal es el caso del criterio de sustentabilidad, el cual ha sido confundido desde sus inicios como sostenibilidad debido en parte a un error intencionado en su traducción, en el seno de las Naciones Unidas, desde el idioma inglés (Sustentability). Darle cualidades y características del objeto y del sujeto a la vivienda, tales como forma y comportamiento responsables, es posible si logramos, mediante

3 Según Izquierdo (1999), el omnijeto es el campo relacional sujeto-objeto. Según Gómez (1999), el territorio debe ser entendido como omnijeto (la vida y sus procesos, poblaciones humanas y no humanas), en un fluir y permanecer trascendente. una técnica interpretativa, como la hermenéutica diatópica, entender, (y sobre todo aceptar entender), al objeto como sujeto y viceversa, dando origen a una especie de "animismo científico" contemporáneo, lo cual nos permitiría entender, no solamente lo que nuestros ancestros y hermanos indígenas han entendido como "madre naturaleza", durante siglos; sino también, a la naturaleza misma como nosotros mismos y de esta manera restaurar la noción original de responsabilidad.

Atributos humanos como la dignidad y la responsabilidad, podrían ser explicados, entendidos y transformados como atributos de la vivienda humana. Por otro lado, atributos del objeto como la resiliencia, podrían ser explicados, entendidos y transformados en el sujeto responsable.

De esta manera, resiliencia, dignidad y responsabilidad serían los nuevos criterios para definir la sustentabilidad, tanto de la forma como en el comportamiento de los seres humanos, y por supuesto, de su vivienda.

Las bases de un nuevo paradigma requeridas para tal fin, requieren ser explicadas, entendidas y transformadas a un nivel dimensional, o mejor, multidimensional más complejo, (no por ello más complicado), que nos permita apre- 
ciar la realidad tridimensional desde una consciencia cuadridimensional. Es por esto, que la antigua tríadica, atribuida, (erróneamente o no) a Vitruvio, es explicada, entendida y transformada como el tetraedro de (alguien).

\section{Sustentabilidad de la Forma}

Desde los tiempos de Marco Vitruvio Polión (S. I. a. C), la sustentabilidad de la forma fue entendida como firmitas, la solidez, durabilidad y estabilidad de una forma construida como medio para mantener o sostener los designios ideales: utilitas y venustas.

Según Vásquez (1995), es falso que Vitruvio dijera que las bases de la utilización y/o función de la arquitectura, entendida como un todo, descansan en tres principios: la Belleza (Venustas), la Firmeza (Firmitas) y la Utilidad (Utilitas), ya que al leer el tratado constatamos que esos tres elementos los cita el autor como referencia exclusiva a los edificios públicos, y particularmente, a ciertos edificios públicos que hoy en día entenderíamos como de equipamientos.

Si aceptamos (y sobre todo si comprobamos), lo anterior, debemos entonces preguntarnos: ¿Además de la a los equipamientos, cuáles serían las bases contemporáneas para "otras" manifestaciones o creaciones arquitectónicas, tales como la vivienda, el espacio público e incluso, el paisaje como producto cultural?

Sin embargo, es más sencillo y cuesta menos esfuerzo intelectual, entender la tríada vitruviana como una síntesis de toda la arquitectura, tal y como quedó establecido tras la publicación de un resumen de la obra por Perrault (1673), constituyéndose en una auténtica delusión repetida por generaciones de arquitectos.

\section{Sustentabilidad del Comportamiento}

La concepción mecanicista de un universo inmutable y determinado nos obligaba a entender "sustentar" como "sostener" el "status quo".

Alrededor del siglo XVII se gesta una visión material de la realidad que entiende los objetos a partir de sus propiedades primarias o geométricas, esto es, de su forma, tamaño, magnitud, disposición y movimiento. Esta filosofía denominada mecánica o corpuscular orienta de manera decisiva los desarrollos del quehacer científico y filosófico a partir de ese período. Aunque lo más decisivo estaba por venir: a partir de 
esa manera de entender los fenómenos físicos y biológicos, se pasa también a inferir razonamientos de tipo psicológico que experimentaron su influencia y extensión a las ciencias denominadas sociales o humanas.

Es a partir de Descartes (1650), en lo que a su física concierne, que se construye la concepción moderna del mundo natural y a partir de ahí se derivan teorías hacia lo social, lo cívico y lo civilizado, como base de un comportamiento urbano: la urbanidad.

El comportamiento ciudadano ideal "era" el de un personaje determinado por un rol específico y sujeto a leyes universales que buscan el "bien común". La cultura ciudadana como causa y efecto de una ciudad "educadora" aún forma parte de los ideales de una política pública "modernizadora".

\section{Dignidad del sujeto}

La dignidad es un atributo humano y como tal, es una idea que ha venido evolucionando históricamente desde la época de los grandes imperios que intentaron, todos ellos a su manera, fundar una civilización. Antiguamente, en el imperio Romano (27 a.C. - 476 d.C.), quien tenía dignidad era solamente el ciudadano, quien habitaba la Civitas.
El ser humano era considerado como persona (quien caracteriza un personaje), solamente si gozaba de ciertos atributos, entre ellos la dignidad, junto con la potestad, la libertad, la ciudadanía y el pater-familis o sui-juris romano. Dichos atributos le otorgaban al sujeto una total aptitud para ser titular de derechos y contraer obligaciones o deberes y curiosamente también había un requisito al nacer: tener una forma humana.

Al evolucionar el imperio, el estatus de ciudadanía fue ampliado a los peregrinos (forasteros libres) y a los romanos que no residían en la ciudad. Finalmente, se introdujo una discriminación de índole religioso que determinaba que los herejes no pudiesen contraer matrimonio, ostentar títulos ni votar. Este último requisito fue introducido en la cultura Romana cuando Constantino I el Grande (c. 274-337), emperador romano (306-337), se convirtió al cristianismo y nombró esa religión como culto oficial.

En todo caso, la dignidad fue y sigue aun siendo la cualidad de un sujeto «valioso». Actualmente, el humano es valioso sólo por ser humano, aunque dicha idea oculta un sesgo que restringe y otorga derechos y deberes: siempre y cuando sea un humano «racional» y capaz de decidir «autónomamente». 
Supuestamente la dignidad debe ir acompañada de la «libertad» y del «poder creador» y el sujeto «tiende» a «progresar» y «medrar» por su naturaleza «divina». Actualmente, es un tema clave en la Bioética que se encuentra estrechamente relacionado con la capacidad de sustentabilidad del sujeto.

\section{Forma y Comportamiento ante un nuevo paradigma}

Si bien el término griego paradigma (parádeigma), se refiere a que existe una postura epistemológica determinada dependiendo del lugar desde donde yo asuma un juicio y en todo caso, implica que se le considere un modelo a seguir. Actualmente urge configurar un nuevo paradigma que oriente a la humanidad hacia la sustentabilidad, tanto de la especie humana como de todos los seres vivos.

En un medio ambiente "vivo" caracterizado por un desorden aparente, la imperfección de la forma y el comportamiento ineficiente de los seres vivos forma parte del nuevo criterio de sustentabilidad: autorregulación, diversidad y dinamismo.

La sustentabilidad ya no es un atributo exclusivo de la forma y el compor- fundamentalmente una característica del sujeto que es capaz de sustentarse a sí mismo y a los demás, del ser humano digno.

\section{Es así como "RESTITUTAS" et} DIGNITAS", se constituyen en los componentes que le hacían falta a la tríadica (vitruviana o no), para llegar a ser constructos o mentefactos tetraédricos que ayuden a constituir la nueva realidad paradigmática que procura la nueva civilización humana, basada esta vez en principios que garanticen, de una vez por todas, que todos podamos medrar y vivir como nos merecemos de aquí al futuro.

Resiliencia y Dignidad son los nuevos criterios para definir la sustentabilidad, tanto de la forma como en el comportamiento de los seres humanos.

4 El término Restitutas, en este contexto significa un sujeto de naturaleza emotiva y perceptiva que se expresa por medio de la investigación y la comprensión, que ama la libertad de movimiento, de pensamiento y de vida y le gusta sentirse deseado. Por otro lado, su naturaleza expresiva s es consistente, ya que se expresa en la línea recta, la atención al detalle, la seguridad de que ama lo que afirma y confirma, la propiedad y la ley que ampara. Tiene un talento natural, ya que es una mente de pensamiento desbordado, que se expresa como pensador inspirado que eleva las ideas y hace de cada idealización una realización. Se destaca en labores que requieren de la comunión de su pensamiento con la cosa pensada, en labores más bien cerebrales que manuales ya que ama las cosas del pensamiento, más al crearlas que al disfrutarlas. Siendo profesional dedicado al arte y la arquitectura podría destacar en una labor comunitaria, sirviendo al público 
Tanto el objeto como el sujeto pueden ser entendidos como omnijeto: la resiliencia y la dignidad que posibilitan la sustentabilidad en la relación objeto-sujeto.

La omnijetividad, según Adolfo Izquierdo Uribe (n. 1948), es una realidad compleja en la cual no hay sujeto ni objeto sin interdependencia entre ambos. Es un campo relacional, en virtud al cual, es posible pensar una alternatividad a la idea convencional de desarrollo, como por ejemplo: la felicidad.

\section{Bases para un nuevo paradigma.}

La dignidad es un concepto normalmente atribuido al sujeto, pero mediante un cambio de enfoque es posible entender que un objeto puede también ser digno, en la medida en que pueda sustentarse.

La resiliencia es una idea atribuida al objeto, que mediante una nueva mirada puede entenderse como una característica del nuevo sujeto sustentable.

En un intento de actualizar el legado vitruviano y superar esa antigua delusión que separa y confunde forma y comportamiento, objeto y sujeto, etc., hemos diseñado tres tetraedros cuyas bases continúan siendo la tradicional triádica venustas, firmitas y utilitas, y cuyos vértices son virtualitas (verosimilitud), restitutas (resiliencia) y dignitas (dignidad), como soporte al hipercubo o cubo tetradimensional definido como sustentabilidad del campo relacional objeto-sujeto-omnijeto.

De todo lo anterior, vamos a desarrollar un poco más la idea de resiliencia y un poco menos la idea de verosimilitud, para lograr equilibrar mejor la idea de dignidad en la vivienda, la cual es el objetivo final del presente escrito.

\section{¿Qué es la Resiliencia?}

La resiliencia, tanto de los sistemas como de las personas, (tomando prestados términos de la sociología y la ecología), es la capacidad para mantener, tanto el propósito fundamental como la integridad ante circunstancias que han cambiado dramáticamente.

La resiliencia es la necesaria idea contemporánea de sustentabilidad, toda vez que éste último concepto ha envejecido y ha sido tan manipulado que carece ya de sentido. 
Según Zolli y Healy (2012), quienes han intentado entender la respuesta a la sencilla pregunta de ¿Por qué será que las cosas vuelven en últimas a su lugar?, la inestabilidad se ha convertido en la norma que rige todos los aspectos de la vida contemporánea, hasta el punto en que nuestras sociedades han vivido cambios profundos en lo económico, social, político y medioambiental, entre muchos aspectos.

Las transformaciones son únicas, pero en la actualidad no pueden entenderse como eventos aislados, pues todo parece interconectado y relacionado, sin que importen como antes las distancias geográficas y culturales. Este escenario impone nuevos retos para los integrantes de toda empresa, institución o grupo social, pues exige respuestas más rápidas y flexibles a los problemas que surgen en cualquier organización.

La manera para llegar a ellas es manteniendo una actitud innovadora, enfocada en la búsqueda de soluciones sostenibles para todos, un pensamiento que asuma las transformaciones y promueva la acción colaborativa entre las personas; en suma, una capacidad especial para de ella, como se define la resiliencia. De esa forma, los cambios se podrán soportar y prever, evitando consecuencias irreparables.

Aunque similar, la redundancia no implica resiliencia, ya que finalmente reduce la eficiencia del sistema y las copias resultan inútiles cuando la situación cambia dramáticamente.

\section{Patrones de Resiliencia}

La resiliencia puede ser interpretada sistémicamente mediante principios que actúan interdependientemente como patrones de resiliencia:

- Apretados círculos de realimentación.

- Reorganización dinámica.

- Mecanismos internos de defensa.

- Desligamiento.

- Diversidad.

- odularidad.

- Simplicidad.

- Conglomerarse y/o aglomerarse.

Cristopher (936), quien ha desarrollado como arquitecto un "lenguaje de patrones", nos advierte finalmente: "No reinventes la rueda". Los lenguajes de 
patrones se utilizan para formalizar los valores de decisiones cuya efectividad resulta obvia a través de la experiencia, pero que es difícil de documentar y pasar a los aprendices.

También son herramientas útiles a la hora de estructurar el conocimiento y comprender sistemas complejos sin caer en la simplificación extrema.

Así como las palabras deben tener una relación gramática y semántica entre ellas para crear un lenguaje oral útil, los patrones de diseño deben estar relacionados los unos con los otros para poder formar un lenguaje de patrones.

En los primeros trabajos de Alexander (1969), está implícita la idea de que los patrones deben estar organizados en estructuras lógicas o estructuras intuitivas. La estructura (jerárquica, iterativa, etc.) puede variar, dependiendo del tema. Cada patrón debe indicar su relación con otros patrones y con el lenguaje en sí.

\section{¿Y...Cual es el problema de la vivienda "digna"?}

La complejidad de la realidad del hábitat «popular» es tal, que podría considerarse como inconmesurable, al igual que los números irracionales.
La inconmensurabilidad, según la filosofía de la ciencia, es la imposibilidad de comparación de dos teorías cuando no hay un lenguaje teórico común. Si no hay manera de compararlas, entonces no podemos decir cuál es mejor y correcta, y por tanto, no podemos tildar la vivienda de informal o ilegal, solo por el hecho de ser popular.

El asunto se complica según Feyerabend (1975), para quien, el problema es semántico, ya que los términos que usamos se comportan como "falsos amigos" en idiomas que utilizan palabras aparentemente iguales pero con significados distintos.

\section{La Delusión de la Forma}

Los Arquitectos y en especial los Diseñadores y «Artistas», creemos desaforadamente que la forma de nuestras creaciones DEBE ajustarse a un determinado contexto físico y/o ideológico, cuando la evidencia contemporánea demuestra que dicho ajuste, en la realidad, es prácticamente inexistente.

Es por esto que, partiendo de un recuento histórico sobre los métodos que se han usado en el diseño, Alexander (1969), ve la necesidad de 
crear un método verdaderamente científico dado que los existentes no son suficientemente rigurosos.

El problema de los métodos tradicionales es que recurren a términos verbales que corresponden más a una tradición cultural que a la estructura real del problema. Por eso, la clave se encuentra en el análisis riguroso del problema y en adaptar a éste la estructura del programa del diseño y no al revés.

Si bien es cierto que en todo problema de diseño existen dos componentes: uno formado por exigencias fuera del control del diseñador y otro por la forma que el diseñador debe adaptar a dichas exigencias, siempre acaba prevaleciendo el lenguaje de patrones que se configura de manera inconsciente, es decir, sin el diseñador, aunque sea incluso para hacer que el problema se transforme en otro nuevo.

\section{¿Quées una Delusión?}

Un autor anónimo en la Internet nos regaló una interesante definición del término: "una persistente falsa creencia mantenida frente a fuerte evidencia contradictoria, especialmente como un síntoma de desorden psiquiátrico"
(Dawkin, 1941). Lo más parecido a una religión, y la arquitectura, para muchos, se ha convertido en una auténtica religión: dogmática, adoratriz, ciega.

A continuación, Dawkins (1941), en su libro "El Espejismo de Dios", (2006), nos invita a escapar de dicha trampa, al retarnos con la imaginación:

Imagine; junto a John Lennon, un mundo sin religión. Imagine que no existen terroristas suicidas; que no existió el 11 de septiembre, ni el 7 de julio, y que no existieron ni las cruzadas ni las cacerías de brujas, ni el Complot de la Pólvora, ni la partición india, ni las guerras palestinas / israelitas, ni las masacres Serbias / Croatas / musulmanas, ni persecución de judíos como "asesinos de Cristo", ni "problemas" en Irlanda del Norte, ni "asesinatos por honor", ni evangelistas televisivos de trajes brillantes y de abombados peinados estafándole su dinero a las personas crédulas ("Dios quiere que usted dé hasta que duela"). Imagine que no existieron los talibanes que explotaron las estatuas antiguas, ni decapitaciones públicas de blasfemos, ni azotes sobre pieles femeninas por el crimen de mostrar unos centímetros de ella. 
La Hermeneútica Diatópica como antídoto a la Delusión Arquitectónica.

La delusión arquitectónica ante la inconmensurabilidad de la realidad consiste en hacer creer que es posible lograr un ajuste eficiente y eficaz entre la forma y su contexto.

La hermeneútica diatópica consiste en la comprensión mutua (entre diferentes) de diferentes «universos de sentido»: entender cómo se entienden las cosas en lugares distintos antes de entender las cosas de manera descontextualizada.

Boaventura (1940), se refiere a lo anterior, en términos de una traición ocasionada por la traducción de conceptos entre culturas intentando encontrar un significado en común, cuando lo que habría que hacer sería simplemente reconocer que somos diferentes y que no vamos a ponernos de acuerdo nunca aunque lo aparentemos en la forma.

El derecho a una vivienda digna se basa en criterios interculturales de derechos humanos fundamentales que deberían ser reinterpretados diatópicamente.

Sin embargo, en la práctica, los eufemismos confunden lo ético con lo estético, lo bueno con lo bonito y no dejan entender la verdadera raíz del conflicto.

\section{¿Y cuál es la forma de la vivienda "digna"?}

Logrando el ajuste entre los constructos tetraédricos anteriormente mencionados, conseguimos dar forma a una vivienda digna, cuyos valores Éticos se equiparan a sus criterios Estéticos; en asentamientos cuya norma de comportamiento corresponden a una misma forma de emplazamiento, y cuyo compromiso Urbano - Arquitectónico basado en lo Ético es el mismo compromiso Arquitectónico - Urbano (Estético).

\section{Vivienda digna.}

En términos interculturales diatópicos la dignidad es un atributo de la vivienda en el lugar donde se vive entendido en términos de quienes lo viven.

La dignidad del lugar donde se vive supone una mediación topofílica (afectiva) entre la psiquis humana y el ethos (modo de ser) del lugar habitado.

El apego o amor por el lugar y el espíritu protector del lugar (genius loci), son ideas precursoras del nuevo 
paradigma. Tanto Yori (1884) como Bachelard (1962), ambos se basan en Heidegger (2003), para redescubrir en últimas que no todas las construcciones son moradas, ni todo morar es habitar, ya que la vivienda no puede reducirse a un simple fenómeno de residir o de "estar". En idioma Misak, vivienda significa literalmente: "donde voy viviendo", en una especie de presente eterno y continuo que se extiende desde la casa al vecindario, al espacio laboral, lúdico, religioso o místico, sea este privado o público.

Los valores que suponen la dignidad de la morada del humano (la naturaleza) son la armonía y la diversidad entendidas bajo criterios culturales (normas de conducta).

Vivienda digna no debe ser entendida como irracional, informal solamente porque $\mathrm{NO}$ somos capaces de entender al OTRO yo mismo.

Hábitat «popular» es realmente un hábitat «emergente» y una vivienda "generatriz» en una ciudad en movimiento. Según Ascher (2004), la ciudad es nuestra y está en movimiento y según Harvey (1935), tenemos derecho a la ciudad. Ambos autores coinciden desde aproximaciones diferentes, el primero desde la hipermodernidad, el segundo desde la añeja geografía política marxista, en que no podemos seguir mirando el espacio y el tiempo por separado sin entender el movimiento, y en que no podemos continuar analizando la causa y el efecto de los fenómenos sin entender el cambio. A continuación, algunas frases sueltas, a manera de consideraciones para ser discutidas de manera diatópica:

\section{¿Acaso la dignidad tiene forma?}

- La dignidad es un criterio humano.

- La forma es natural.

- El humano es natural.

- La forma «digna» es un criterio humano.

- La Estética es un criterio CULTURAL

\section{Dignidad y Derechos Humanos}

- La dignidad es un derecho humano.

- El humano merece dignidad SIEMPRE.

- La vivienda DIGNA es un derecho de TODOS los humanos.

Finalmente, pongámonos de acuerdo entonces en definir claramente aquello en lo que NO estamos de acuerdo. 


\section{Conclusiones}

No estamos de acuerdo.

- Con creer que las creencias son suficiente argumento para justificar nuestras creencias.

- Con justificar que la dignidad humana está por debajo de la religión, el poder y el dinero.

- Con creer que la "vivienda digna" es un derecho humano fundamental sin entender qué es "vivienda digna" y qué es "derecho humano fundamental" como tal.

- En considerar que la robustez es sinónimo de resiliencia. Un sistema robusto puede ser increíblemente frágil cuando se trata de reconstruirse a sí mismo luego de su destrucción total o parcial.

- En creer que los sistemas resilientes sean infalibles, todo lo contrario: las fallas habituales y a escala manejable son necesarias para mantener reorganizados y limpios los sistemas.

- En considerar la resiliencia como sinónimo de racionalidad, formalidad y orden. Como lo es la vida, igualmente la resiliencia implica desorden, imperfección e ineficiencia, e incluso hasta la muerte.

\section{Referencias}

Alexander, C. (1969). Ensayo sobre la Síntesis de la Forma. Buenos Aires: Eds. Infinito.

Ascher, F. (2004). Los nuevos princípios del urbanismo. Madrid: Alianza Editorial.

Bachelard, G. (1962). La Poétique de l'espace". México: Fondo de Cultura Económica, .

Boaventura De Sousa, Santos. (1940), A gramática do tempo: para uma nova cultura política, Volume IV da coleção: Para um novo senso comum. São Paulo: Ed. Cortez.

Dawkins, R. (1941). El Espejismo de Dios. Nueva York: Bantan Press.

Descartes, R. (1650). Oeuvres Philosophiques. Paris: Éditions Classiques Garnier, Bordas.

Feyerabend, P. (1975). Against Method: Outline of an Anarchistic Theory of Knowledge. En M. Radner \& S. Winokur, eds., Analyses of Theories and Methods of Physics and Psychology. Minneapolis: University of Minnesota Press.

Gomez, A. (1999). El territorio urbano regional de cara al nuevo milenio: Trayectorias y Perspectivas. Bitácora Urbano/Territorial, 1(4), (pp. $21-25$ ). 
Harvey, D. (1935). Ciudades rebeldes: Del derecho a la ciudad a la revolución urbana. Madrid: Ed. Akal.

Heidegger, M. (2003). Bemerkungen zu Kunst-Plastik-Raum - Die Kunst und der Raum. Pamplona: Universidad Pública de Navarra.

Izquierdo, A. (1999). Espacio Temporalidad y Omnijetividad - Una Aproximación Epistemológica. Nómadas, 1(11). 241 - 248.
Perrault, C. (1673). Orden de los cinco tipos de columnas según el método de los antiguos. Recuperado de http://d2aohiyo3d3idm. cloudfront.net/publications/virtuallibrary/0892362332.pdf

Yori, C. (1999). Topofilia o la dimensión poética del habitar. Bogotá DC.: Pontificia Universidad Javeriana.

Zolli, A. y Healy, A. (2012). Resiliencia: Por qué las cosas vuelven a su lugar. Bogotá: Editorial Norma. 